$63^{\text {ème }}$ Congrès de la SFCO, 03014 (2015)

DOI:10.1051/sfco/20156303014

(C) Owned by the authors, published by EDP Sciences, 2015

\title{
Lymphomes non hodgkinien primaires de la cavité orale : à propos de deux localisations maxillaires
}

\author{
Farouk M, Kissi L, Ben Yahya I \\ Faculté de médecine dentaire de Casablanca, Rue Abou Al Alaa Zahar 21100, BP. 9157 Mers Sultan, \\ Casablanca, Casablanca, Maroc
}

Les lymphomes sont des tumeurs malignes des lignées cellulaires de lymphocytes. Ils sont principalement classés comme lymphome de Hodgkin, et non hodgkinien (Tanaka 2012).

Le lymphome non hodgkinien (LNH) provient de l'évolution maligne et de la prolifération de cellules lymphoïdes de type B (Patte 2010).

Le lymphome primaire non hodgkinien se produit généralement dans les ganglions lymphatiques, mais 20 à $30 \%$ des sites peuvent êtresextra-nodaux. La cavité orale comme site extranodale primaire est très rare (Vinoth 2012).

Les sujets de sexe masculin sont les plus touchés par cette tumeur (Freitas 2008).

Les localisations les plus fréquentes sont au niveau du plais mou et la gencive, d'autres localisations sont plus rares comme la région périapicale et la base de la langue. Cliniquement la lésio se présente sous forme d'une tuméfaction indolore, associée ou non à une mobilité dentaire (Vinoth 2012).

Le diagnostic est confirmé par l'examen anatomopathologique. L'immuno-phénotypage complète le diagnostic en identifiant la présence des marqueurs B. La polychimiothérapie constitue actuellement le centre du traitement du fait de la forte chimio sensibilité et de la médiocre radiosensibilité de la tumeur. La survie globale des stades localisés est proche de 100 \% (Chbicheb 2011).

Ce travail rapporte deux cas de (LNH) primaires de la cavité orale avec deux localisations maxillaires différents.

Le premier cas clinique : une localisation palatine.

Il s'agit d'un homme âgé de 45 ans, qui est venu consulter pour une asymétrie faciale droite. L'examen clinique a montré la présence d'une tuméfaction molle et indolore à la palpation, au niveau de la région maxillaire droite s'étendant vers le palais mou.

Les examens radiologiques (panoramique et cônebeam) ont montré une ostéolyse étendue à l'hémimaxillaire droit.

Une biopsie a été réalisé dont l'examen anatomopathologique et immunohistochimique ont confirmé le diagnostic du LNH.

Le patient a été adressé au service d’hématologie, un traitement à base de chimiothérapie a été instauré.

Les contrôles cliniques et radiologiques après une année du traitement ont montré une cicatrisation parfaite du site et une rémission complète.

Deuxième cas clinique : une localisation péri-apicale.

Il s'agit d'un homme âgé de 52 ans, dont le motif de consultation était une gène masticatoire due à une mobilité importante de la 14 et 15.

L'examen radiologique a montré une lyse osseuse profonde en regard de ces dents.

L'extraction des prémolaires a été faite.

This is an Open Access article distributed under the terms of the Creative Commons Attribution License 4.0, which permits unrestricted use, distribution, and reproduction in any medium, provided the original work is properly cited. 
En peropératoire la présence le curetage périapicale a ramené un tissu d’allure pathologique qui a été envoyée pour examen anatomopathologique dont le résultat était un LNH.

Le patient a été adressé au service d'hématologie pour prise en charge.

Le but de ce travail est de montrer le rôle du médecin dentiste dans le dépistage des pathologies tumorales malignes.

Nom et adresse du conférencier

Mohammed FAROUK

Faculté de médecine dentaire de Casablanca

Rue Abou Al Alaa Zahar 21100, BP. 9157 Mers Sultan Casablanca

Casablanca (Maroc)

mohammed.dentiste@gmail.com 